Alma Mater Studiorum - Università di Bologna DEPARTMENT OF ECONOMICS

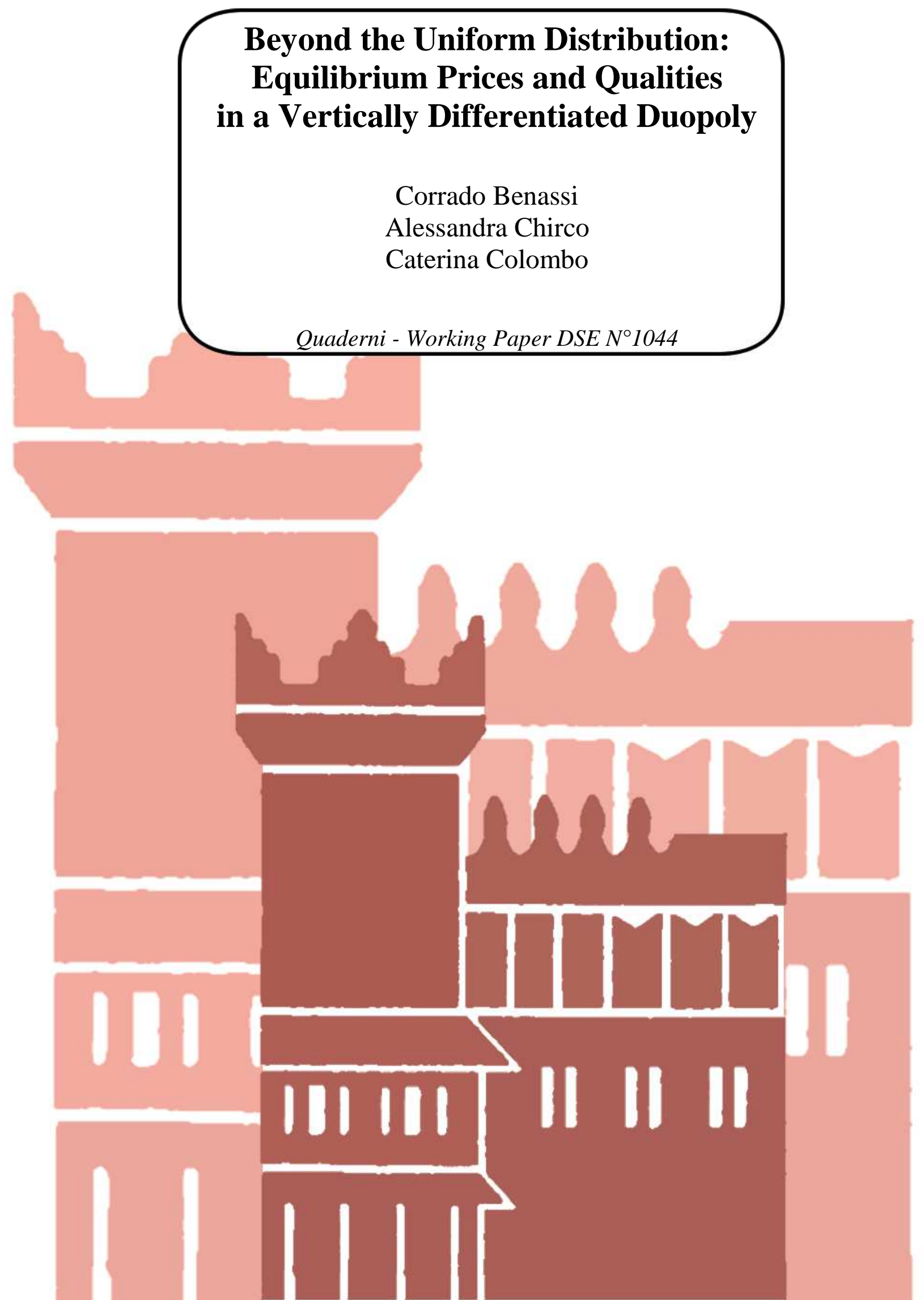


December 2015

\title{
Beyond the Uniform Distribution: Equilibrium Prices and Qualities in a Vertically Differentiated Duopoly
}

\author{
Corrado Benassi* \\ Alessandra Chirco \# \\ Caterina Colombo $\S$
}

* Corresponding Author. Dipartimento di Scienze Economiche, Alma Mater Studiorum - Università di Bologna, Piazza Scaravilli 2, 40122 Bologna, Italy. corrado.benassi@unibo.it

\# Dipartimento di Scienze dell'Economia, Università del Salento, via per Monteroni, 73100 Lecce, Italy. alessandra.chirco@unisalento.it

$\S$ Dipartimento di Economia e Management, Università degli Studi di Ferrara, via Voltapaletto 11, 44121 Ferrara, Italy. caterina.colombo@unife.it

ABSTRACT. The paper proves the existence of a subgame perfect Nash equilibrium in a vertically differentiated duopoly with uncovered market, for a large set of symmetric and asymmetric distributions of consumers, including, among others, all logconcave distributions. The proof relies on the 'income share elasticity' representation of the consumers' density function, which ensures the analytical tractability of the firms' optimality conditions at a high level of generality. Some illustrative examples of the solution are offered, in order to assess the impact of distributive shocks on the equilibrium market configuration.

KEYwords. Vertical differentiation, duopoly, non-uniform distribution, subgame perfect equilibrium, income share elasticity.

JEL Codes: L13, L11, D43, C72 


\section{Introduction}

There are two main reasons for products to be differentiated. The first is that consumers love variety, so that product differentiation is the market response to a property of individual preferences. The second is that consumers are different from each other, and product differentiation is the proper market response to this diversity. In these two explanations, agents' heterogeneity plays quite a different role. Indeed, in the modern literature the key reference for the former is the Dixit and Stiglitz (1977) model of monopolistic competition, which is based on a representative agent assumption - though recent extensions of their basic framework allowed to capture the effects of consumers' heterogeneity on several aspects of market competitiveness (see, among others, Benassi et al, 2005; Osharin et al,2014; Bertoletti and Etro, 2015). By contrast, within the approach based on consumers' diversity, the representation of agents' heterogeneity, along with the properties of preferences, is the key building block of any model. Actually, it is the shape of the distribution of consumers which defines the market environment faced by firms - the distribution of consumers in the space of the product characteristics within horizontal differentiation, the distribution of the willingness to pay for quality, which can be taken as a proxy for income distribution, under vertical differentiation. ${ }^{1}$ As a consequence, any sound theoretical representation must be robust to a wide range of assumptions about the functional form of the consumers' distribution - a necessary condition to ensure realism and predictive power.

This objective has already been attained in the case of horizontal differentiation. Indeed, the well known paper by Anderson et al. (1997) identifies a set of conditions on the distribution of consumers' along the 'linear city' which ensure that the subgame perfect equilibrium in prices and locations exists and is unique. For vertical differentiation, however, the state of the art is far less satisfactory - which is in a way surprising, given the economic and social relevance of the involved heterogeneity, namely income heterogeneity. As shown in the same paper by Anderson et al, there is indeed an isomorphism between the linear city and the vertical differentiation models, which allows for an extension of their generalization to the latter framework; but this occurs only under the assumption of full market coverage, i.e. when consumers split between firms producing goods of different quality levels, but are all 'rich enough' to buy at least the lowest quality good at

\footnotetext{
${ }^{1}$ The key insight along these lines was presented in the seminal papers by Gabszewitz and Thisse $(1979,1980)$.
} 
the equilibrium prices. However, the isomorphism does not apply to the uncovered market case, where some consumers may be 'too poor' to buy at the equilibrium prices, so that market segmentation includes market exclusion.

For vertically differentiated markets exhibiting this essential feature, the discussion of the role of agents' heterogeneity has offered only partial or preliminary results. By using the Mussa and Rosen (1978) specification of the indirect utility function, the existence of a duopolistic equilibrium in prices has been proved by Furth (2011) under logconcave densities. The full solution has been developed only for two specific densities: Yurko (2011) develops an algorithm to solve numerically a oligopoly model of the ShakedSutton type (Shaked and Sutton, 1982) under a lognormal distribution of consumers, while Benassi et al. (2006) offer an analytical solution of the price-quality game under a trapezoid distribution - clearly, for models of vertical differentiation with uncovered market there is still an open problem in terms of robustness, manageability and empirical relevance.

This paper aims at offering an effective contribution along these three lines. On the one hand, it generalizes the solution of the duopoly model with vertical differentiation and uncovered market for a wide set of consumers' distributions. It proves the existence of the subgame perfect price-quality equilibrium for a range of symmetric and asymmetric distributions, which includes - but is not limited to - all logconcave distributions. On the other hand, our proof makes use of simple but powerful analytical tools which allow us to deal with the complexity of the analytics and to develop a manageable solution procedure, which can be applied to the whole set of the admissible distributions. Finally, the possibility to obtain explicit solutions for different configurations of the parameters of the same distribution makes it possible to analyse how relevant distributive shocks might affect the market prices, the quality spread, as well as the profitability and the market shares of the two firms.

We are able to prove the existence of equilibrium at the aforementioned degree of generality by uncovering a key feature of the model, namely that the optimality conditions at the two stage of the game can be seen as a block-recursive system of equations. Block-recursiveness ensures that the equilibrium market shares of the two firms are determined, under appropriate constraints, by the optimal behavior of the low quality firm at the two stages, while the optimal behavior of the high quality firm determines, given those shares, the equilibrium levels of qualities. The block-recursiveness property shows up once the density function describing the consumers' income distribution is represented through the so-called income share elasticity (Esteban, 1986), an analytical tool which has already proved to be extremely 
useful in the analysis of the relation between consumers' heterogeneity and market structure, whenever market demand is intrinsecally shaped by the distributon itself (e.g., Benassi et al, 2002).

The usefulness of Esteban's formulation goes beyond the existence proof. By clearly bringing out the above block-recursiveness property, it has the additional advantage of simplifying considerably the actual computation of the equilibrium solutions. Indeed, we provide some applications of our solution procedure, by analyzing the effects of some distributive shocks in two illustrative cases with a symmetric and an asymmetric consumers' distribution. The regularities which emerge from our examples suggest that both for symmetric and asymmetric distributions, a reduction in inequality is associated with an increase in the quality spread, in the overall market coverage, and in the market share and profitability of the high quality firm. Interestingly enough, the threshold level of income required to enter the market increases, while that required to afford the high quality may either increase or decrease.

In the sequel of the paper we proceed as follows. The basic framework is set in Section 2. In Section 3 we prove the existence and uniqueness of the equilibrium in prices. Section 4 is devoted to proving the existence of the subgame perfect equilibrium in prices and quality. The effects of distributive shocks on the equilibrium solution are discussed through some examples in Section 5, while in Section 6 we gather some conclusions.

\section{The basic framework}

We consider a duopolistic market for a vertically differentiated product in which two firms play a simultaneous two-stage game with respect to prices and qualities. At the first stage firms set the quality of their product, $s \in$ $\left(0, s_{\max }\right]$. We denote with $H$ the firm choosing the higher quality $s_{H}$, and with $L$ the firm choosing the lower quality $s_{L}$. Once the qualities have been set, firms produce at a cost independent of $s$ and normalized to zero. At the second stage firms compete with respect to prices, respectively $p_{H}$ and $p_{L}$. The game is solved by backward induction.

\subsection{Preferences and demand}

In this market demand stems from a continuum of heterogeneous consumers, whose size is normalized to 1 . We assume that the generic consumer $j$ 's utility function is of the Mussa and Rosen (1978) type: 


$$
\begin{array}{ll}
U_{j}=\theta_{j} s-p, & \text { if she purchases one unit of a good of quality } s \\
U_{j}=0, & \text { if she does not purchase. }
\end{array}
$$

Consumers differ across $\theta$, the preference parameter which denotes the willingness to pay for quality. According to a standard interpretation (Tirole 1988, p.97), $\theta_{j}$ can also be seen as an index of the consumer $j$ 's 'income', so that consumers' heterogeneity can be interpreted as an income heterogeneity, described by the distribution of $\theta$. In the sequel, we assume that $\theta$ is distributed according to a continuously differentiable density function $f(\theta)$ defined over the support $\left[0, \theta_{\max }\right]$, and we denote with $F(\theta)=\int_{0}^{\theta} f(\tau) d \tau$ the corresponding cumulative distribution function.

Given the above utility function, consumer $j$ enters the market and buys one unit of the good only if $\theta_{j}$ is greater than the hedonic price $(p / s)$ set by at least one of the two firms. If $p_{L} / s_{L}<p_{H} / s_{H}$, so that both firms are active in the market, ${ }^{2}$ it is possible to define $\theta_{L} \equiv p_{L} / s_{L}$ as (the income of) the consumer which is indifferent between buying from $L$ and not buying, and $\theta_{H} \equiv\left(p_{H}-p_{L}\right) /\left(s_{H}-s_{L}\right)$ as (the income of) the consumer which is indifferent between buying from $L$ and buying from $H$. Therefore, the demand functions faced by firm $L$ and $H$ are respectively:

$$
\begin{aligned}
D_{L} & =\int_{\theta_{L}}^{\theta_{H}} f(\theta) d \theta=F\left(\theta_{H}\right)-F\left(\theta_{L}\right) \\
D_{H} & =\int_{\theta_{H}}^{\theta_{\max }} f(\theta) d \theta=1-F\left(\theta_{H}\right)
\end{aligned}
$$

\subsection{The distribution of $\theta$}

In the above setting, the distribution of $\theta$ is the key element shaping market demand and therefore the properties of the firms' market interactions. In order to preserve a high degree of generality, we do not set any definite functional form for this 'distribution of income', but we simply assume that $f(\theta)$ satisfies the following general conditions:

Condition 1. $f(\theta)>0$ for all $\theta \in\left(0, \theta_{\max }\right)$

\footnotetext{
${ }^{2}$ Our hypothesis on the support of the distribution will actually ensure that at equilibrium (a) both firms are active in the market, and (b) the market is uncovered (i.e. there is a lower tail of the distribution of $\theta$ at which consumers do not buy the good). For a discussion of this point in the case of uniform distribution, see Wauthy (1996).
} 
Condition 2. $\eta(\theta) \equiv \frac{\theta f(\theta)}{1-F(\theta)}$ is increasing in $\theta$ for all $\theta \in\left(0, \theta_{\max }\right)$, with $\lim _{\theta \rightarrow \theta_{\max }} \eta(\theta)>1$.

Condition 3. $\frac{1}{1-F(\theta)}$ is convex in $\theta$ for all $\theta \in\left(0, \theta_{\max }\right)$.

Notice that $1-F(\theta)$ can be seen as the share of active (buying) consumers (i.e. the degree of market coverage or overall market demand) if the hedonic price $p / s=\theta$ is the lowest hedonic price available in the market. Conditions 2 and 3 can therefore be interpreted as reasonable restrictions on the shape of this peculiar demand curve. Since $\eta(\theta)$ is the absolute value of the elasticity of $1-F(\theta)$, Condition 2 can be seen as ruling out that the elasticity of market demand might be constant or decreasing in the hedonic price. This condition is indeed satisfied for most of the commonly used distributions (e.g. normal, lognormal, Beta, etc.), with the notable exception of the Pareto distribution, which generates a function $1-F(\theta)$ with constant elasticity. Condition 3 imposes that the $1-F(\theta)$ function be not too convex, which is generally (and also here) required for the profit functions to be concave. Notice that Conditions 2 and 3 are satisfied by all $f$ distributions (including the logconcave) which generate logconcave $1-F$ functions; the latter, however, are only a subset of the admissible distributions (see An, 1998).

The following properties will be useful in the sequel.

Remark 1. For a given $f(\theta)$, consider the following function, known as income share elasticity (Esteban, 1986):

$$
\pi(\theta)=1+\frac{\theta f^{\prime}(\theta)}{f(\theta)}
$$

By means of the $\pi(\theta)$ function, it is possible to write Condition 2 as:

$$
\eta(\theta)+\pi(\theta)>0 \text { for all } \theta \in\left(0, \theta_{\max }\right)
$$

Moreover, it can be checked that Condition 3 boils down to:

$$
2 \eta(\theta)+\pi(\theta)>1 \text { for all } \theta \in\left(0, \theta_{\max }\right)
$$

Notice that logconcavity of the $1-F(\theta)$ function would imply $\eta(\theta)+\pi(\theta)>$ 1 , which is more restrictive of both Condition 2 and Condition 3.

Should $\theta$ be strictly interpreted as income, the function $\pi$ would measure the relative marginal change in the share of income accruing to class 
$\theta$, brought about by a marginal increase in $\theta$; in general, Esteban (1986) shows that a one-to-one relationship exists between the $\pi$ function and the underlying density $f .{ }^{3}$ Representing Conditions 2 and 3 in terms of $\eta$ and $\pi$ is relevant in our framework, since this elasticity formulation remarkably contributes to the analytical tractability of the two-stage game.

\section{The Price Stage of the Game}

At the price stage of the game firms compete with respect to prices, for given qualities $s_{H}$ and $s_{L}$. Given the profit functions:

$$
\begin{aligned}
\Pi_{H}\left(p_{H}, p_{L} ; s_{H}, s_{L}\right) & =p_{H}\left(1-F\left(\theta_{H}\right)\right) \\
\Pi_{L}\left(p_{H}, p_{L} ; s_{H}, s_{L}\right) & =p_{L}\left(F\left(\theta_{H}\right)-F\left(\theta_{L}\right)\right)
\end{aligned}
$$

the first order conditions (FOCs) for firm $H$ and $L$ are respectively:

$$
\begin{aligned}
& \frac{\partial \Pi_{H}}{\partial p_{H}}=1-F\left(\theta_{H}\right)-\frac{p_{H}}{\Delta} f\left(\theta_{H}\right)=0 \\
& \frac{\partial \Pi_{L}}{\partial p_{L}}=F\left(\theta_{H}\right)-F\left(\theta_{L}\right)-p_{L}\left(\frac{f\left(\theta_{H}\right)}{\Delta}+\frac{f\left(\theta_{L}\right)}{s_{L}}\right)=0
\end{aligned}
$$

where $\Delta \equiv s_{H}-s_{L}$. Consider first the FOC of firm $H$. By defining $\varphi_{H} \equiv$ $\left(\partial \theta_{H} / \partial p_{H}\right)\left(p_{H} / \theta_{H}\right)=p_{H} /\left(p_{H}-p_{L}\right)>1$, equation (1) can be rewritten as:

$$
1-F\left(\theta_{H}\right)-\varphi_{H} \theta_{H} f\left(\theta_{H}\right)=0
$$

and, using the definition of $\eta(\theta)$, as

$$
\eta\left(\theta_{H}\right) \varphi_{H}=1
$$

Equation (3) states the standard condition that with zero costs, the FOC of firm $H$ requires that (in absolute value) the elasticity of its demand with respect to its price - given by the product of the elasticity of its demand with respect to $\theta_{H}$ and the elasticity of $\theta_{H}$ with respect to $p_{H}$ - be equal to 1 . The key implication of equation (3) is that whenever it holds, firm $H$ sets a price such that $\theta_{H}$ takes a value at which $\eta\left(\theta_{H}\right)<1$.

\footnotetext{
${ }^{3}$ It should be noticed that the $\pi$-formulation of the density often allows simpler representations of the relevant features of the distribution. E.g., $\pi(\theta)=1$ identifies the modal value of $\theta$, while "the Pareto, Gamma and Normal density functions correspond to constant, linear and quadratic elasticities, respectively" (Esteban, 1986, p.442).
} 
Consider now the FOC of firm $L$. By defining $\varphi_{L} \equiv\left(\partial \theta_{H} / \partial p_{L}\right)\left(p_{L} / \theta_{H}\right)=$ $-p_{L} /\left(p_{H}-p_{L}\right)=1-\varphi_{H}<0$, and using again the definition of $\eta(\theta)$, equation (2) can be formulated as:

$$
\eta\left(\theta_{L}\right) \frac{1-F\left(\theta_{L}\right)}{F\left(\theta_{H}\right)-F\left(\theta_{L}\right)}-\eta\left(\theta_{H}\right) \varphi_{L} \frac{1-F\left(\theta_{H}\right)}{F\left(\theta_{H}\right)-F\left(\theta_{L}\right)}=1
$$

Also for firm $L$ the FOC implies that the absolute value of its demand elasticity with respect to $p_{L}$ be equal to $1 .{ }^{4}$ In the sequel it will be useful to refer to the following reformulation of equation (4):

$$
\left(1-\varphi_{L} \eta\left(\theta_{H}\right)\right)\left(1-F\left(\theta_{H}\right)\right)-\left(1-F\left(\theta_{L}\right)\right)\left(1-\eta\left(\theta_{L}\right)\right)=0
$$

\subsection{Existence of equilibrium at the price stage}

For a given pair $\left(s_{L}, s_{H}\right)$, an equilibrium at the price stage is a pair $\left(\theta_{L}^{*}, \theta_{H}^{*}\right)$ satisfying (3) and (5), at which the Second Order Conditions (SOCs) for profit maximization are verified. Clearly, the optimal prices $p_{L}^{*}$ and $p_{H}^{*}$ can then be recovered from the definitions of $\theta_{L}$ and $\theta_{H}$.

In order to prove that under Conditions 1-3 an equilibrium exists, we first introduce the following Lemma.

Lemma 1. For given qualities, equation (3) can be rewritten as:

$$
\theta_{L}=\frac{\Delta}{s_{L}}\left(\frac{1}{\eta\left(\theta_{H}\right)}-1\right) \theta_{H} \equiv h\left(\theta_{H}\right)
$$

with $\theta_{L}=h\left(\theta_{H}\right)$ decreasing in $\theta_{H}$. This function is economically meaningful when defined over the interval $\left[\underline{\theta}_{H}, \bar{\theta}_{H}\right]$, where $\underline{\theta}_{H}$ satisfies $\eta\left(\underline{\theta}_{H}\right)=\Delta / s_{H}$. and $\bar{\theta}_{H}$ satisfies $\eta\left(\bar{\theta}_{H}\right)=1$, so that $0<\underline{\theta}_{H}<\bar{\theta}_{H}<\theta_{\max }$.

Proof. Using the definitions of $\varphi_{H}, \theta_{H}$ and $\theta_{L}$, we can express $\varphi_{H}$ in terms of $\theta_{L}$ and $\theta_{H}$ as:

$$
\varphi_{H}=1+\frac{s_{L}}{\Delta} \frac{\theta_{L}}{\theta_{H}}
$$

\footnotetext{
${ }^{4}$ It is easy to check that equation (4) actually states this condition, once the demand for $L$ is written as $D_{L}=\left(1-F\left(\theta_{L}\right)\right)-\left(1-F\left(\theta_{H}\right)\right)$. Notice that $\eta\left(\theta_{L}\right)$ is the $p_{L}$ elasticity of overall market demand - the elasticity of demand with respect to $\theta$ evaluated at $\theta_{L}$ multiplied by the (unit) elasticity of $\theta_{L}$ with respect to $p_{L}-$ while $\eta\left(\theta_{H}\right) \varphi_{L}$ is the $p_{L}$ elasticity of the demand accruing to $H$ - the elasticity of $1-F(\theta)$ evaluated at $\theta_{H}$, multiplied by the elasticity of $\theta_{H}$ with respect to $p_{L}$.
} 
Equation (3') can then be obtained by substituting the above expression into (3), and solving for $\theta_{L}$. Notice that (3') restates the FOC of firm $H$ at the price stage in terms of $\theta_{L}$ and $\theta_{H}$ only. For it to be economically meaningful, it must satisfy the following restrictions: (i) $\theta_{L} \leq \theta_{H}$, and (ii) $\theta_{L} \geq 0$. Restriction (i) defines the lower bound of the value of $\eta\left(\theta_{H}\right)$ along the domain of $\left(3^{\prime}\right)$; indeed, the following inequality:

$$
\frac{\Delta}{s_{L}}\left(\frac{1}{\eta\left(\theta_{H}\right)}-1\right) \theta_{H} \leq \theta_{H}
$$

requires

$$
\eta\left(\theta_{H}\right) \geq \frac{\Delta}{s_{H}}
$$

Restriction (ii) defines the upper bound of $\eta\left(\theta_{H}\right)$ along the domain of (3'). The following inequality:

$$
\frac{\Delta}{s_{L}}\left(\frac{1}{\eta\left(\theta_{H}\right)}-1\right) \theta_{H} \geq 0
$$

requires

$$
\eta\left(\theta_{H}\right) \leq 1
$$

Given Condition 2, the boundary equalities $\eta\left(\theta_{H}\right)=\Delta / s_{H}$ and $\eta\left(\theta_{H}\right)=1$ implicitly define the boundary values $\underline{\theta}_{H}$ and $\bar{\theta}_{H}$ stated in the Lemma. Since $\eta(0)=0$ and $\lim _{\theta \rightarrow \theta_{\max }} \eta(\theta)>1,0<\underline{\theta}_{H}<\bar{\theta}_{H}<\theta_{\max }$. Finally, by differentiating (3') we get:

$$
h^{\prime}\left(\theta_{H}\right)=\frac{\Delta}{s_{L}} \frac{1}{\eta\left(\theta_{H}\right)}\left(1-\pi\left(\theta_{H}\right)-2 \eta\left(\theta_{H}\right)\right)
$$

which implies that

$$
\operatorname{sign}\left\{h^{\prime}\left(\theta_{H}\right)\right\}=\operatorname{sign}\left\{1-\pi\left(\theta_{H}\right)-2 \eta\left(\theta_{H}\right)\right\}
$$

Therefore, along (3') $\theta_{L}=h\left(\theta_{H}\right)$ is decreasing if Condition 3 holds.

We can now prove the following Proposition.

Proposition 1. If Conditions 1-3 are satisfied, there exists an equilibrium at the price stage for any given pair $\left(s_{L}, s_{H}\right)$.

Proof. At equilibrium both (3) and (5) must be satisfied. By using $\varphi_{L}=$ $1-\varphi_{H}$ and by substituting for $\varphi_{H}$ from (3), equation (5) can be rewritten as:

$$
\left(2-\eta\left(\theta_{H}\right)\right)\left(1-F\left(\theta_{H}\right)\right)-\left(1-F\left(\theta_{L}\right)\right)\left(1-\eta\left(\theta_{L}\right)\right)=0
$$


Using now for $\theta_{L}$ the $h\left(\theta_{H}\right)$ function defined in (3'), an equilibrium exists, if there exists a value $\theta_{H}^{*} \in\left[\underline{\theta}_{H}, \bar{\theta}_{H}\right]$ at which:

$$
\left(2-\eta\left(\theta_{H}^{*}\right)\right)\left(1-F\left(\theta_{H}^{*}\right)\right)-\left(1-F\left(h\left(\theta_{H}^{*}\right)\right)\right)\left(1-\eta\left(h\left(\theta_{H}^{*}\right)\right)\right)=0
$$

and the SOCs are satisfied at $\theta_{H}^{*}$ and $\theta_{L}^{*}=h\left(\theta_{H}^{*}\right)$. Define now the continuous function:

$$
\lambda\left(\theta_{H}\right) \equiv\left(2-\eta\left(\theta_{H}\right)\right)\left(1-F\left(\theta_{H}\right)\right)-\left(1-F\left(h\left(\theta_{H}\right)\right)\right)\left(1-\eta\left(h\left(\theta_{H}\right)\right)\right)
$$

where $\theta_{H} \in\left[\underline{\theta}_{H}, \bar{\theta}_{H}\right]$. Since,

$$
\lambda\left(\underline{\theta}_{H}\right)=1-F\left(\underline{\theta}_{H}\right)>0
$$

and

$$
\lambda\left(\bar{\theta}_{H}\right)=-F\left(\bar{\theta}_{H}\right)<0
$$

continuity of $\lambda\left(\theta_{H}\right)$ implies there exists a value $\theta_{H}^{*} \in\left(\underline{\theta}_{H}, \bar{\theta}_{H}\right)$ such that $\lambda\left(\theta_{H}^{*}\right)=0$. Given $\theta_{H}^{*}$ and $\theta_{L}^{*}=h\left(\theta_{H}^{*}\right)$, and given $s_{L}$ and $s_{H}$, the corresponding prices $p_{L}^{*}$ and $p_{H}^{*}$ can be obtained. The latter are equilibrium solutions of the price stage, if the SOCs of firms $H$ and $L$ are satisfied at $\theta_{H}^{*}$ and $\theta_{L}^{*}$. This is indeed the case, as verified in Appendix A.

\subsection{Uniqueness of the equilibrium at the price stage}

We recall that a necessary condition for a two-stage game to have a solution is that equilibrium at the second stage be unique. With respect to the above equilibrium in prices, we now prove the following Proposition.

Proposition 2. If Conditions 1-3 hold, the equilibrium at the price stage is unique.

Proof. The equilibrium at the price stage is unique if $\lambda\left(\theta_{H}^{*}\right)=0$ implies that the first order derivative $\lambda^{\prime}(\cdot)$ is negative at $\theta_{H}^{*}$. By evaluating the derivative of (6) at $\theta_{H}^{*}$ we obtain

$$
\begin{aligned}
\lambda^{\prime}\left(\theta_{H}^{*}\right)= & -\left(1-F\left(\theta_{H}^{*}\right)\right) \frac{\eta\left(\theta_{H}^{*}\right)}{\theta_{H}^{*}}\left(\pi\left(\theta_{H}^{*}\right)+2\right)+ \\
& +h^{\prime}\left(\theta_{H}^{*}\right)\left(1-F\left(\theta_{L}^{*}\right)\right) \frac{\eta\left(\theta_{L}^{*}\right)}{\theta_{L}^{*}}\left(\pi\left(\theta_{L}^{*}\right)+1\right)
\end{aligned}
$$


Under Conditions 1-3, Lemma 1 ensures that $h^{\prime}\left(\theta_{H}^{*}\right)<0$. Moreover, according to Condition $2, \pi(\theta)>-\eta(\theta)$. Since at equilibrium $\eta\left(\theta_{L}^{*}\right)<\eta\left(\theta_{H}^{*}\right)<1$, it follows that $\lambda^{\prime}\left(\theta_{H}^{*}\right)<0$.

For future reference, we conclude our discussion of the price stage by collecting in the following Lemma the results of some comparative statics exercises on the reaction of the optimal prices to changes in the quality levels.

Lemma 2. At the price stage equilibrium the price of firm $H$ is decreasing in $s_{L}$ (i.e. $d p_{H}^{*} / d s_{L}<0$ ), while the price of firm $L$ is increasing in $s_{H}$ (i.e. $\left.d p_{L}^{*} / d s_{H}=d p_{L}^{*} / d \Delta>0\right)$. Moreover, $d p_{L}^{*} / d s_{L}-\theta_{L}^{*}<0$.

Proof. See Appendix B.

\section{The subgame perfect equilibrium}

As discussed in Section 3, our price equilibrium is defined in terms of a pair of indifferent consumers, $\left(\theta_{H}^{*}, \theta_{L}^{*}\right)$, which, given qualities, obviously deliver the firms' optimal prices. When dealing with the two-stage game, the device of solving the model in terms of the $\theta$ 's turns out to be particularly useful. Indeed, a subgame perfect equilibrium can be defined as a pair of indifferent consumers and a pair of quality levels, such that both the optimality conditions with respect to prices for given qualities, and the optimality conditions with respect to quality - which take into account the effect of quality on optimal prices - are satisfied. Again, prices can be recovered residually from the definitions of $\theta_{H}$ and $\theta_{L}$. In subsection 4.1 we first derive the firms' FOCs at the quality stage, and we show that they can be expressed exclusively in terms of $\theta_{H}$ and $\theta_{L}$. In subsection 4.2 we prove that a subgame perfect equilibrium exists under Conditions 1-3.

\subsection{The quality stage}

At the first stage of the game the derivatives of the firms' profits with respect to quality can be written as:

$$
\frac{\partial \Pi_{H}}{\partial s_{H}}=\left(1-F\left(\theta_{H}\right)\right) p_{H H}-p_{H} f\left(\theta_{H}\right)\left(\frac{\partial \theta_{H}}{\partial p_{H}} p_{H H}+\frac{\partial \theta_{H}}{\partial p_{L}} p_{L H}+\frac{\partial \theta_{H}}{\partial s_{H}}\right)
$$




$$
\begin{aligned}
\frac{\partial \Pi_{L}}{\partial s_{L}}= & p_{L L}\left[F\left(\theta_{H}\right)-F\left(\theta_{L}\right)\right]+p_{L} f\left(\theta_{H}\right)\left(\frac{\partial \theta_{H}}{\partial p_{H}} p_{H L}+\frac{\partial \theta_{H}}{\partial p_{L}} p_{L L}+\frac{\partial \theta_{H}}{\partial s_{L}}\right)+ \\
& -p_{L} f\left(\theta_{L}\right)\left(\frac{\partial \theta_{L}}{\partial p_{L}} p_{L L}+\frac{\partial \theta_{L}}{\partial s_{L}}\right)
\end{aligned}
$$

where $p_{H H} \equiv \partial p_{H}^{*} / \partial s_{H}, p_{H L} \equiv \partial p_{H}^{*} / \partial s_{L}, p_{L H} \equiv \partial p_{L}^{*} / \partial s_{H}$, and $p_{L L} \equiv$ $\partial p_{L}^{*} / \partial s_{L}$. All these derivatives of prices with respect to qualities can be obtained by total differentiation of the solution of the price stage, as in Lemma 2.

Consider first firm $H$. Collecting $p_{H H}$ and using (1), equation (7) can be rewritten as

$$
\frac{\partial \Pi_{H}}{\partial s_{H}}=\frac{p_{H} f\left(\theta_{H}\right)}{\Delta}\left(p_{L H}+\theta_{H}\right)=\frac{\theta_{H} f\left(\theta_{H}\right)}{\eta\left(\theta_{H}\right)}\left(p_{L H}+\theta_{H}\right)
$$

The above expression is positive, since $p_{L H}>0$ according to Lemma 2 . The profits of firm $H$ being monotonically increasing in $s_{H}$ implies that at the quality stage firm $H$ chooses:

$$
s_{H}^{* *}=s_{\max }
$$

Equation (9) confirms in our general setting the results obtained in the literature under explicit formulations of the distribution of $\theta$, according to which the quality set by firm $H$ is independent of that chosen by firm $L$ and, in the absence of costs for quality, coincides with highest quality $s_{\max }$.

Consider now firm $L$. Collecting $p_{L L}$ and using (2), equation (8) can be rewritten as:

$$
\frac{\partial \Pi_{L}}{\partial s_{L}}=\frac{p_{L}}{\Delta} f\left(\theta_{H}\right)\left(p_{H L}+\theta_{H}\right)+f\left(\theta_{L}\right) \theta_{L}^{2}
$$

so that, using the definition of $\eta$ and equation (1), we obtain the following FOC for profit maximization of firm $L$ :

$$
\theta_{H}\left(\frac{1}{\eta\left(\theta_{H}\right)}-1\right) f\left(\theta_{H}\right)\left(p_{H L}+\theta_{H}\right)+f\left(\theta_{L}\right) \theta_{L}^{2}=0
$$

Using again the definition of $\eta$ and equation (5'), we can restate this condition as

$$
\left(1-\eta\left(\theta_{H}\right)\right)\left(p_{H L}\left(\theta_{H}, \theta_{L}\right)+\theta_{H}\right)+\frac{2-\eta\left(\theta_{H}\right)}{1-\eta\left(\theta_{L}\right)} \eta\left(\theta_{L}\right) \theta_{L}=0
$$

where we recall that $p_{H L}$ is a function of $\theta_{H}$ and $\theta_{L}$ only, as in equation (B.2) of Appendix B, and is negative according to Lemma 2. 


\subsection{Existence of a subgame perfect equilibrium}

We recall that equations (3) and (5) identify the necessary conditions for optimal price strategies given qualities, in terms of the corresponding income of the indifferent consumers $\theta_{H}$ and $\theta_{L}$. As to quality decisions, taking into account the effect of quality on the optimal prices, equation (9) identifies the optimal quality of firm $H$, while equation (10) can be seen as the implicit relation between $\theta_{H}$ and $\theta_{L}$ required by the necessary condition for optimization with respect to quality of firm $L$. Therefore, a subgame perfect equilibrium in prices and qualities can be defined in terms of a pair of indifferent consumers $\left(\theta_{H}^{* *}, \theta_{L}^{* *}\right)$ and a pair of quality levels $\left(s_{H}^{* *}, s_{L}^{* *}\right)$ such that equations (3), (5), (9) and (10) hold, and the SOCs for profit maximization are satisfied. Again, the optimal prices can be derived from the definitions of $\theta_{H}$ and $\theta_{L}$.

Indeed, the system of equations (3), (5), (9) and (10) exhibits very useful properties. First, we recall that equation (5), after substitution of (3) for $\varphi_{L}=1-\varphi_{H}$, can be reformulated as in equation $\left(5^{\prime}\right)$, repeated here for convenience:

$$
\left(2-\eta\left(\theta_{H}\right)\right)\left(1-F\left(\theta_{H}\right)\right)=\left(1-\eta\left(\theta_{L}\right)\right)\left(1-F\left(\theta_{L}\right)\right)
$$

Rewriting then (3) as in (3'), the system (3'), (5'), (9) and (10) clearly exhibits recursiveness. Equation (9) defines $s_{H}^{* *}$ independently of all other variables. Equations (5') and (10) are a subsystem in terms of $\theta_{H}$ and $\theta_{L}$ only, while equation (3') determines $s_{L}$ for given $s_{H}, \theta_{H}$ and $\theta_{L}$. This recursiveness reflects an important economic property of the model: provided that an equilibrium exists, the market shares of the two fims are determined by the price and quality FOCs of firm $L$, under the constraint imposed by equation (3) that at the equilibrium value of $\theta_{H}$ the elasticity of the market share of firm $H$ with respect to price must be equal to $1 / \eta\left(\theta_{H}\right)$.

Before studying the solution of the above recursive system, we introduce the following Lemma.

Lemma 3. Equation (5') defines an implicit relation $\theta_{L}=g\left(\theta_{H}\right)$, which is increasing in $\theta_{H}$. This relation is economically meaningful when defined over the interval $\left[\theta_{H}^{\text {low }}, \theta_{H}^{\text {up }}\right]$ - where $\theta_{H}^{\text {low }}$ satisfies $\left(2-\eta\left(\theta_{H}\right)\right)\left(1-F\left(\theta_{H}\right)\right)=1$ and $\theta_{H}^{u p}$ satisfies $\eta\left(\theta_{H}^{u p}\right)=1$.

Proof. By implicit differentiation, the elasticity of $\theta_{L}$ with respect to $\theta_{H}$ along $(5 ')$ is

$$
g^{\prime}\left(\theta_{H}\right) \frac{\theta_{H}}{\theta_{L}}=\frac{\left(1-F\left(\theta_{H}\right)\right)}{\left(1-F\left(\theta_{L}\right)\right)} \frac{\eta\left(\theta_{H}\right)}{\eta\left(\theta_{L}\right)} \frac{\left(2+\pi\left(\theta_{H}\right)\right)}{\left(1+\pi\left(\theta_{L}\right)\right)}
$$


which is positive under Condition 2 , given that at equilibrium $\eta\left(\theta_{H}\right)<1$. As in the case of the $h(\bullet)$ function in Lemma 1 , for the $g(\bullet)$ function to be economically meaningful, the relation it describes between $\theta_{H}$ and $\theta_{L}$ must satisfy two restrictions: (i) $\theta_{L}=g\left(\theta_{H}\right) \geq 0$; (ii) $\theta_{L}=g\left(\theta_{H}\right) \leq \theta_{H}$. Along $g(\bullet)$, restriction (i) is satisfied for all $\theta_{H} \geq \theta_{H}^{\text {low }}$, where $\theta_{H}^{\text {low }}>0$ solves $\left(5^{\prime}\right)$ when $\theta_{L}=0$, i.e. solves $\left(2-\eta\left(\theta_{H}^{\text {low }}\right)\right)\left(1-F\left(\theta_{H}^{\text {low }}\right)\right)=1$. However, for the domain of the $g(\bullet)$ function to be consistent with the domain of the $h(\bullet)$ function, equation $\left(5^{\prime}\right)$ must be defined for those values of $\theta_{H}$ at which the condition $h\left(\theta_{H}\right) \geq 0$ is satisfied along (3'). This occurs for $\theta_{H} \leq \theta_{H}^{u p}$ where $\theta_{H}^{u p}$ satisfies $\eta\left(\theta_{H}^{u p}\right)=1$. The check that $\theta_{H}^{\text {low }}<\theta_{H}^{\text {up }}$ is straightforward. Indeed, $\left(2-\eta\left(\theta_{H}^{\text {low }}\right)\right)\left(1-F\left(\theta_{H}^{\text {low }}\right)\right)=1$ implies $\eta\left(\theta_{H}^{\text {low }}\right)=\left(1-2 F\left(\theta_{H}^{\text {low }}\right)\right) /\left(1-F\left(\theta_{H}^{\text {low }}\right)\right)$. The latter is positive by definition of $\eta$, and lower than 1 . Condition 2 then ensures $\theta_{H}^{\text {low }}<\theta_{H}^{u p}$ Consider now restriction (ii). It is easy to check that $\theta_{H}=\theta_{L}$ cannot occur along the $g(\bullet)$ function. Since the latter is increasing in $\theta_{H}$ with $\theta_{H}^{\text {low }}>0$, this implies that $\theta_{L}<\theta_{H}$ over the domain $\left[\theta_{H}^{l o w}, \theta_{H}^{u p}\right]$.

Given Lemma 3, we now prove the following Proposition:

Proposition 3. If Conditions 1-3 hold, there exists a subgame perfect equilibrium of the price-quality game.

Proof. To prove the above Proposition we proceed by steps. At the first step we prove that the subsystem (5') and (10) in terms of $\theta_{H}$ and $\theta_{L}$ has a solution $\left(\theta_{H}^{* *}, \theta_{L}^{* *}\right)$. At the second step we show that, given $\theta_{H}^{* *}$ and $\theta_{L}^{* *}$ and $s_{H}^{* *}=s_{\max }$, equation (3') delivers an optimal quality level $s_{L}^{* *} \in\left(0, s_{\max }\right)$. Finally, we check that the SOCs for profit maximization are satisfied at that solution.

STEP I. Define the following continuous function:

$$
\begin{aligned}
& \Psi\left(\theta_{H}\right)=\psi\left(\theta_{H}, g\left(\theta_{H}\right)\right)= \\
& =\left(1-\eta\left(\theta_{H}\right)\right)\left(p_{H L}\left(\theta_{H}, g\left(\theta_{H}\right)\right)+\theta_{H}\right)+\frac{2-\eta\left(\theta_{H}\right)}{1-\eta\left(g\left(\theta_{H}\right)\right)} \eta\left(g\left(\theta_{H}\right)\right) g\left(\theta_{H}\right)
\end{aligned}
$$

The above expression is equation (10) where we have used for $\theta_{L}$ the $g\left(\theta_{H}\right)$ implicit function from $\left(5^{\prime}\right)$, defined over the interval $\left[\theta_{H}^{l o w}, \theta_{H}^{u p}\right]$. A pair $\left(\theta_{H}^{* *}, \theta_{L}^{* *}\right)$ solving $\left(5^{\prime}\right)$ and $(10)$ exists, if there exists a $\theta_{H}^{* *}$ such that $\Psi\left(\theta_{H}^{* *}\right)=0$. 
Using the expression for $p_{H L}$ given in equation (B.2) in Appendix B, and recalling equation (3), it can be checked that:

$$
\Psi\left(\theta_{H}^{l o w}\right)=\theta_{H}^{l o w}\left(1-\eta\left(\theta_{H}^{l o w}\right)\right)\left(1-\frac{1}{\eta\left(\theta_{H}^{l o w}\right)}\right)<0
$$

and that

$$
\Psi\left(\theta_{H}^{u p}\right)=\frac{\eta\left(g\left(\theta_{H}^{u p}\right)\right) g\left(\theta_{H}^{u p}\right)}{1-\eta\left(g\left(\theta_{H}^{u p}\right)\right)}>0
$$

Therefore, continuity of $\Psi\left(\theta_{H}\right)$ implies that there exists a $\theta_{H}^{* *}$ such that $\Psi\left(\theta_{H}^{* *}\right)=0$, with $\Psi^{\prime}\left(\theta_{H}^{* *}\right)>0$. Given $\theta_{H}^{* *}$, the corresponding value of $\theta_{L}$ is $\theta_{L}^{* *}=g\left(\theta_{H}^{* *}\right)$.

SteP II. Substituting $s_{H}^{* *}=s_{\max }$ and the pair $\left(\theta_{H}^{* *}, \theta_{L}^{* *}\right)$ into $\left(3^{\prime}\right)$ we obtain

$$
\theta_{L}^{* *}=\frac{s_{\max }-s_{L}}{s_{L}}\left(\frac{1}{\eta\left(\theta_{H}^{* *}\right)}-1\right) \theta_{H}^{* *}
$$

i.e.

$$
\frac{s_{\max }}{s_{L}}=\frac{\theta_{L}^{* *}}{\theta_{H}^{* *}} \frac{\eta\left(\theta_{H}^{* *}\right)}{1-\eta\left(\theta_{H}^{* *}\right)}+1
$$

which, solved for $s_{L}$, implies that $0<s_{L}^{* *}<s_{\max }$.

STEP III. Given $\left(\theta_{H}^{* *}, \theta_{L}^{* *}\right)$ from (5') and (10), and $s_{L}^{* *}$ from (12), we have now to verify whether the SOCs are satisfied at this solution. We have already proved in Appendix A that the SOCs for profit maximization with respect to prices are satisfied for any quality pair. Therefore they are satisfied at $\left(s_{L}^{* *}, s_{\max }\right)$. Given that at the quality stage firm $H$ chooses a corner solution, we need simply to check that the SOC for profit maximization with respect to quality is verified for firm $L$. This is proved in Appendix C.

Proposition 3 establishes that the duopoly model of vertical differentiation with uncovered market has a solution under a wide range of symmetric and asymmetric distributions, which includes - but is not limited to - all logconcave distributions. Besides its theoretical relevance, we believe that this result may have significant implications in terms of our understanding of actual market behaviour. We have shown that, given a specific density function $f(\theta)$, the solution procedure is in principle quite simple, in that it makes use of the associated $\eta(\theta)$ and $\pi(\theta)$ functions only. This provides a useful instrument for the analysis of the effects of distributive phenomena on the configurations of markets with vertically differentiated products. We offer some examples in the sequel. 


\section{Applications: Beta and Dagum distributions}

In this section we explicitly solve our duopoly model assuming that the consumers' $\theta$ s are distributed according to different parametrizations of the symmetric Beta and the Dagum distributions. The former seems relevant as a generalization of the uniform distribution standardly used in vertical differentiation models, while the latter is well known to be a fairly good representation of actual (asymmetric) income distributions. Our exercise will focus on the effects of equal-mean, second-order stochastic-dominance shifts of the distribution, as this allows to consider how the firms' equilibrium choices react to a well-defined change in income dispersion. ${ }^{5}$

The density of the Beta symmetric distribution over the unit interval $[0,1]$ is given by

$$
f(\theta, \gamma)=B(\gamma) \theta^{\gamma-1}(1-\theta)^{\gamma-1}
$$

where $B(\gamma)$ is the Beta function, ${ }^{6}$ and $\gamma \geq 1$ is an index of second-order stochastic dominance for given mean, $\mu=1 / 2$ - accordingly, higher $\gamma$ distributions will Lorenz-dominate lower $\gamma$ distributions. By solving our blockrecursive system, we can calculate the equilibrium values of different relevant variables associated to different values of $\gamma$ : having fixed $s_{H}^{*}=s_{\max }=1$, we report in Table 1 our results for $\gamma=2, \gamma=3$ and $\gamma=4$.

The same exercise can be performed for the Dagum distribution, which in our case one can conveniently write as

$$
g(\theta, \delta)=2 \theta\left(1+\theta^{\delta} K(\delta)\right)^{-\frac{2+\delta}{\delta}} M(\delta)
$$

where the average income has been normalized to 1 , and $\delta>1$ is a concentration parameter such that higher $\delta$ distributions stochastically dominate lower $\delta$ distribution in the second order sense; $K(\cdot)$ and $M(\cdot)$ are constant parameters depending on $\delta$ itself. ${ }^{7}$ The system of the firms' FOC can be solved numerically under different values of the concentration parameter. For $s_{H}^{*}=s_{\max }=1$ we find the results reported in Table 2 for $\delta=2, \delta=3$ and $\delta=4$.

\footnotetext{
${ }^{5}$ Second-order stochastic dominance is well known to have noteworthy normative implications in terms of inequality rankings. In particular, equal-mean, second-order stochastic dominance amounts to Lorenz dominance (Atkinson, 1970).

${ }^{6}$ The Beta function with parameters $(p, q)$ is given by $B(p, q)=\int_{0}^{1} u^{p-1}(1-u)^{q-1} d u$; symmetry requires $p=q=\gamma$. On the Beta distribution see Johnson et al (1995, ch.25). It is easily seen that $\gamma=1$ delivers the uniform distribution

${ }^{7}$ In particular, $K(\delta)=\Gamma^{-\delta}(2 / \delta) \Gamma^{\delta}(3 / \delta) \Gamma^{\delta}((\delta-1) / \delta)$ and $M(\delta)=\Gamma^{2}((\delta-1) / \delta)$ $\Gamma^{2}(3 / \delta) / \Gamma^{2}(2 / \delta)$, where $\Gamma(\cdot)$ is the Gamma functon such that $\Gamma(\delta)=\int_{0}^{\infty} e^{-z} z^{\delta-1} d z$. On the Dagum distribution and its properties see Kleiber (2008).
} 
Table 1: Values of relevant variables under different concentration parameters: the Beta distribution

\begin{tabular}{|c|c|c|c|}
\hline & $\gamma=2$ & $\gamma=3$ & $\gamma=4$ \\
\hline$\theta_{H}^{* *}$ & 0.36725 & 0.35902 & 0.35900 \\
\hline$\theta_{L}^{* *}$ & 0.12398 & 0.13087 & 0.13878 \\
\hline $1-F\left(\theta_{H}^{* *}\right)$ & 0.69445 & 0.75066 & 0.78506 \\
\hline$F\left(\theta_{H}^{* *}\right)-F\left(\theta_{L}^{* *}\right)$ & 0.26325 & 0.23110 & 0.2058 \\
\hline$p_{H}^{* *}$ & 0.24235 & 0.25307 & 0.26615 \\
\hline$p_{L}^{* *}$ & $6.3655 \times 10^{-2}$ & $6.0776 \times 10^{-2}$ & $5.8513 \times 10^{-2}$ \\
\hline$s_{H}^{* *}-s_{L}^{* *}$ & 0.48657 & 0.5356 & 0.57838 \\
\hline$\prod_{H}^{* *}$ & 0.16830 & 0.18997 & 0.20894 \\
\hline$\Pi_{L}^{* *}$ & $1.6757 \times 10^{-2}$ & $1.4045 \times 10^{-2}$ & $1.2042 \times 10^{-2}$ \\
\hline$\Pi_{H}^{* *} / \Pi_{L}^{* *}$ & 10.044 & 13.526 & 17.351 \\
\hline
\end{tabular}

Table 2: Values of relevant variables under different concentration parameters: the Dagum distribution

\begin{tabular}{|lllll|}
\hline & $\delta=2$ & & $\delta=3$ & \\
\cline { 2 - 2 }$\theta_{H}^{* *}$ & 0.48336 & & 0.59110 & \\
$\theta_{L}^{* *}$ & 0.17222 & & 0.20747 & \\
$1-F\left(\theta_{H}^{* *}\right)$ & 0.63433 & & 0.69171 & 0.22412 \\
$F\left(\theta_{H}^{* *}\right)-F\left(\theta_{L}^{* *}\right)$ & 0.29748 & & 0.2655 & 0.71092 \\
$p_{H}^{* *}$ & 0.32542 & & 0.39859 & 0.25305 \\
$p_{L}^{* *}$ & $8.7424 \times 10^{-2}$ & 0.10411 & 0.43754 \\
$s_{H}^{* *}-s_{L}^{* *}$ & 0.49237 & & 0.4982 & 0.50275 \\
$\Pi_{H}^{* *}$ & 0.20642 & & 0.27571 & 0.31106 \\
$\Pi_{L}^{* *}$ & $2.6007 \times 10^{-2}$ & $2.7641 \times 10^{-2}$ & $2.8200 \times 10^{-2}$ \\
$\Pi_{H}^{* *} / \Pi_{L}^{* *}$ & 7.9371 & 9.9747 & 11.03 \\
\hline
\end{tabular}

The numbers considered in Tables 1 and 2, though clearly limited to very specific cases, lend themselves to some observations. In our exercise asymmetry does not seem to play a relevant role. The pattern of most variables is indeed the same under both distributions, i.e. irrespective of symmetry. In both cases an increase in income concentration leads to widening the degree of market coverage and narrowing the market for low quality goods - and 
hence to a larger market for high quality commodities. In both cases the quality spectrum gets larger as income becomes more concentrated. Indeed, at unchanged qualities, lower dispersion implies a positive shift in demand for both firms, making it more convenient an aggressive price behaviour of the high quality firm. The low quality firm protects its market share by enlarging the quality differential, and adjusting its price accordingly. While the equilibrium price of the high quality increases in all our examples, the price of the low quality increases (in the Dagum case) or decreases (in the symmetric Beta case), depending on the balance between the initial demand stimulus and the effect of the reduction of quality.

In both our examples the market share of the high quality firm increases, while that of the low quality one decreases. However, due to the different ways in which the dominance parameter modifies the shape of the two distributions, this pattern of the market shares is accompanied by a decrease of the threshold income to access the high quality market $\left(\theta_{H}^{* *}\right)$ in the case of the Beta distribution, while in the case of the Dagum distribution this income increases. Finally, and in our view most interestingly, under both distributions the minimum level of income required to enter the market $\left(\theta_{L}^{* *}\right)$ increases. The shrinking of the left tail of the distribution decreases the incentive to trade-off the intensive margin on buyers for the extensive margin on excluded consumers. An unpleasant consequence of the reduction of inequality is that there are poor consumers who are driven out of the market by this very reduction.

\section{Conclusions}

The relationship between consumers' heterogeneity, and in particular personal income distribution, and market structure is an intriguing issue in modern economic theory. On the one side, it calls for a renewed attention to the role of market demand in shaping the firms' competitive environment; on the other side it creates a link between economic and social phenomena such as inequality, poverty, or income polarization and several dimensions of the firms' behavior affecting market efficiency. However, there is a key obstacle for a full development of this stream of economic analysis, which concerns its analytical tractability at high levels of generality.

This paper contributes in this direction by offering a general proof for the existence of a subgame perfect equilibrium in pure strategies in a duopolistic model with vertical differentiation and uncovered market. We extend the existing literature by showing that under very mild conditions on the shape 
of income distribution - which are indeed less restrictive than those implied by logconcavity - it is possible to go beyond the proof of existence of a unique equilibrium in prices. By making use of the 'Esteban elasticity' representation of the consumers' density function we are able to prove the existence of a two-stage equilibrium in prices and qualities and to offer a manageable algorithm to actually compute the above solution. Our application of this algorithm to different configurations of the Beta and Dagum distributions envisages the multiplicity of exercises that can in principle be performed to evaluate the effects of distributional changes in our basic setup.

The proof of the existence of equilibrium in a vertically differentiated duopoly for a wide set of density functions is a fundamental step, but it leaves open other interesting questions. Further investigation is required to extend our existence result to an oligopolistic setting and to formulate non densityspecific propositions about the relationship between income dispersion and the number of qualities offered in the market.

\section{References}

[1] An Y. M.(1998), Logconcavity versus Logconvexity: A Complete Characterization, Journal of Economic Theory, vol.80, pp.350-369.

[2] Anderson S.P., Goeree J.K. and Ramer R. (1997), Location, location, location, Journal of Economic Theory, vol.77, pp.102-127

[3] Atkinson A.B. (1970), On the Measurement of Inequality. Journal of Economic Theory, vol.2, pp.244-63.

[4] Benassi C., Chirco A. and Colombo C. (2005), A model of monopolistic competition with personal income dispersion, Metroeconomica, vol.56, pp.305-317.

[5] Benassi C., Chirco A. and Colombo C. (2006), Vertical differentiation and the distribution of income, Bulletin of Economic Research, vol.58, pp.345-367.

[6] Benassi C., Chirco A. and Scrimitore M. (2002) Income concentration and market demand, Oxford Economic Papers, vol.54, pp.584-596.

[7] Bertoletti P. and Etro F. (2015), Monopolistic Competition when Income Matters, Economic Journal, doi: 10.1111/ecoj.12329. 
[8] Bonnisseau J.M. and Lahmandi-Ayed R. (2007), Vertical differentiation with non-uniform consumers' distribution, International Journal of Economic Theory, vol.3, pp.179-190.

[9] Dixit A. and Stiglitz J. (1977), Monopolistic competition and optimum product diversity, American Economic Review, vol.67, pp.297-308.

[10] Esteban J. (1986), Income share elasticity and the size of distribution of income, International economic review, vol.27, pp.439-444.

[11] Furth D. (2011), Duopoly Models with Vertical Product Differentiation, International Game theory Review, vol.13, pp.121-140.

[12] Gabszewicz J.J. and Thisse J.F. (1979), Price competition, quality and income disparities, Journal of Economic Theory, vol.20, pp.340-359.

[13] Gabszewicz J.J. and Thisse J.F. (1980), Entry (and exit) in a differentiated industry, Journal of Economic Theory, vol.22, pp.327-338.

[14] Johnson N. L., Kotz S. and Balakrishnan N. (1995), Continuous Univariate Distributions, New York: Wiley, vol.2.

[15] Kleiber C. (2008), A guide to the Dagum distribution. In D.Chotikapanich (ed.) Modeling Income Distributions and Lorenz Curves, Berlin: Springer, pp. 97-117.

[16] Mussa M. and Rosen S. (1978), Monopoly and Product Quality, Journal of Economic Theory, vol.18, pp.301-317.

[17] Osharin A., Thisse J.F., Ushchev P. and Verbus V. (2014), Monopolistic competition and income dispersion, Economics Letters, vol.122, pp.348352.

[18] Shaked A. and Sutton J. (1982), Relaxing price competition through product differentiation, Review of Economic Studies, vol. XLIX, pp.313

[19] Tirole, J. (1988). The Theory of Industrial Organization, Cambridge, MA, and London: The MIT Press.

[20] Waulthy X. (1996), Quality choice in models of vertical differentiation, Journal of Industrial Economics, vol.44, pp.345-353. 
[21] Yurko A.V. (2011), How does income inequality affect market outcomes in vertical differentiated markets?, International Journal of Industrial Organization, vol.29, pp.493-503.

\section{Appendix A. The Second Order Conditions at the price stage}

In this and in the following Appendices we shall make use of the following simplifying notation: $f_{i} \equiv f\left(\theta_{i}\right), F_{i} \equiv F\left(\theta_{i}\right), \eta_{i} \equiv \eta\left(\theta_{i}\right), \pi_{i} \equiv \pi\left(\theta_{i}\right)$, $i=H, L$.

The SOC of firm $H$ requires:

$$
\frac{\partial^{2} \Pi_{H}}{\partial p_{H}^{2}}=-\frac{1}{\Delta}\left(2 f_{H}+\frac{p_{H}}{\Delta} f_{H}^{\prime}\right)<0
$$

By using (1) and recalling the definitions of $\eta(\theta)$ and $\pi(\theta)$ it can be rewritten as

$$
-\frac{1}{\Delta} \frac{\left(1-F_{H}\right)}{\theta_{H}}\left(2 \eta_{H}+\pi_{H}-1\right)<0
$$

which is indeed the case for all $\theta_{H}>0$, if Condition 3 holds.

The SOC of firm $L$ is satisfied if

$$
\frac{\partial^{2} \Pi_{L}}{\partial p_{L}^{2}}=-2\left(\frac{f_{H}}{\Delta}+\frac{f_{L}}{s_{L}}\right)+p_{L}\left(\frac{f_{H}^{\prime}}{\Delta^{2}}-\frac{f_{L}^{\prime}}{s_{L}^{2}}\right)<0
$$

i.e.

$$
f_{H}+\frac{\Delta}{s_{L}} f_{L}+\frac{1}{2} \frac{p_{L}}{\Delta}\left(f_{H}^{\prime}-\left(\frac{\Delta}{s_{L}}\right)^{2} f_{L}^{\prime}\right)>0
$$

Equation (3) allows to reformulate $\Delta / s_{L}$ and $p_{L} / \Delta$ in terms of $\theta_{H}$ and $\theta_{L}$ only:

$$
\begin{aligned}
\frac{\Delta}{s_{L}} & =\frac{\theta_{L}}{\theta_{H}} \frac{\eta_{H}}{1-\eta_{H}} \\
\frac{p_{L}}{\Delta} & =\frac{\theta_{H}}{\eta_{H}}\left(1-\eta_{H}\right)
\end{aligned}
$$

so that using (5') and the definitions of $\eta(\theta)$ and $\pi(\theta)$, the inequality (A.1) can be rewritten as

$$
\eta_{H}+\frac{1}{2}\left(1-\eta_{H}\right)\left(\pi_{H}-1\right)+\frac{1}{2} \frac{2-\eta_{H}}{1-\eta_{L}} \eta_{L} \frac{\eta_{H}}{1-\eta_{H}}\left(1+\pi_{L}\right)>0
$$


At equilibrium, the first two terms are positive since equation (3) ensures $\eta_{H}<1$ and Condition (3) ensures that $\left(\pi_{H}-1\right)>-2 \eta_{H}$. The last term is positive since Condition (2) and equation (3) imply (a) $\eta_{L}<\eta_{H}<1$, and (b) $\pi_{L}>-\eta_{L}>-1$

\section{Appendix B. The comparative statics of the price stage}

By totally differentiating (1) and (2), we obtain the following linear system:

$$
\left(\begin{array}{cc}
\frac{\partial^{2} \Pi_{H}}{\partial p_{H}^{2}} & \frac{\partial^{2} \Pi_{H}}{\partial p_{H} \partial p_{L}} \\
\frac{\partial^{2} \Pi_{L}}{\partial p_{L} \partial p_{H}} & \frac{\partial^{2} \Pi_{L}}{\partial p_{L}^{2}}
\end{array}\right)\left(\begin{array}{c}
d p_{H} \\
d p_{L}
\end{array}\right)=-\left(\begin{array}{c}
\frac{\partial^{2} \Pi_{H}}{\partial p_{H} \partial \Delta_{s}} \\
\frac{\partial^{2} \Pi_{L}}{\partial p_{L} \partial \Delta_{s}}
\end{array}\right) d \Delta_{s}-\left(\begin{array}{c}
\frac{\partial^{2} \Pi_{H}}{\partial p_{H} \partial s_{L}} \\
\frac{\partial^{2} \Pi_{L}}{\partial p_{L} \partial s_{L}}
\end{array}\right) d s_{L}
$$

where the above derivatives can be written as:

- $\frac{\partial^{2} \Pi_{H}}{\partial p_{H}^{2}}=-2 f_{H} \frac{1}{\Delta}-\frac{p_{H}}{\Delta^{2}} f_{H}^{\prime} ; \frac{\partial^{2} \Pi_{H}}{\partial p_{H} \partial p_{L}}=f_{H} \frac{1}{\Delta}+\frac{p_{H}}{\Delta^{2}} f_{H}^{\prime}$

- $\frac{\partial^{2} \Pi_{L}}{\partial p_{L} \partial p_{H}}=f_{H} \frac{1}{\Delta}-\frac{p_{L}}{\Delta^{2}} f_{H}^{\prime}$

- $\frac{\partial^{2} \Pi_{L}}{\partial p_{L}^{2}}=-\frac{2}{\Delta} f_{H}-\frac{2}{s_{L}} f_{L}+\frac{p_{L}}{\Delta^{2}} f_{H}^{\prime}-f_{L}^{\prime} \frac{\theta_{L}}{s_{L}}$

- $\frac{\partial^{2} \Pi_{H}}{\partial p_{H} \partial \Delta_{s}}=f_{H}\left(\frac{\theta_{H}}{\Delta}+\frac{p_{H}}{\Delta^{2}}\right)+\frac{p_{H}}{\Delta} f_{H}^{\prime} \frac{\theta_{H}}{\Delta}$;

- $\frac{\partial^{2} \Pi_{H}}{\partial p_{H} \partial s_{L}}=-f_{H}\left(\frac{\theta_{H}}{\Delta}+\frac{p_{H}}{\Delta^{2}}\right)-\frac{p_{H}}{\Delta} f_{H}^{\prime} \frac{\theta_{H}}{\Delta}$

- $\frac{\partial^{2} \Pi_{L}}{\partial p_{L} \partial \Delta_{s}}=f_{H}\left(\frac{p_{L}}{\Delta^{2}}-\frac{\theta_{H}}{\Delta}\right)+\frac{p_{L}}{\Delta} f_{H}^{\prime} \frac{\theta_{H}}{\Delta}$

- $\frac{\partial^{2} \Pi_{L}}{\partial p_{L} \partial s_{L}}=f_{H}\left(\frac{\theta_{H}}{\Delta}-\frac{p_{L}}{\Delta^{2}}\right)-\frac{p_{L}}{\Delta} f_{H}^{\prime} \frac{\theta_{H}}{\Delta}+2 \frac{\theta_{L}}{s_{L}} f_{L}+\frac{\theta_{L}^{2}}{s_{L}} f_{L}^{\prime}$

and are evaluated at equilibrium. 
Denoting with $A$ the coefficients matrix, its determinant can be written as:

$$
\begin{aligned}
|A|= & \frac{1}{\Delta^{2}} f_{H}^{2}\left(2+\pi_{H}\right)+ \\
& +\frac{1}{\Delta^{2}}\left\{\frac{\Delta}{s_{L}}\left(\left(1+\pi_{L}\right) f_{L}\right)\left(\left(1+\pi_{H}\right) f_{H}+\frac{p_{L}}{\Delta} f_{H}^{\prime}\right)\right\}
\end{aligned}
$$

Condition 2 ensures that $\left(1+\pi_{H}\right)>0$, provided that $\eta_{H}<1$, which is indeed the case at the price stage equilibrium. Since $\theta_{L}<\theta_{H}$, Condition 2 ensures also that at equilibrium $\eta_{L}<1$ so that $\left(1+\pi_{L}\right)>0$. Therefore $|A|>0$ if:

$$
\left(1+\pi_{H}\right) f_{H}+\frac{p_{L}}{\Delta} f_{H}^{\prime}>0
$$

Recalling equation (A.3), at equilibrium we can reformulate this inequality as

$$
\frac{f_{H}}{\eta_{H}}\left(2 \eta_{H}+\pi_{H}-1\right)>0
$$

which is indeed the case under Condition 3.

We can now perform the following comparative statics exercises.

The effect on $p_{L}^{*}$ of a change in $s_{H}$.

First notice that for given $s_{L}, d p_{L}^{*} / d s_{H}=d p_{L}^{*} / d \Delta$. By applying Cramer's rule:

$$
\frac{d p_{L}^{*}}{d \Delta}=\frac{1}{|A|} \frac{p_{L}}{\Delta^{3}} f_{H}^{2}\left(2+\pi_{H}\right)
$$

where the RHS is evaluated at equilibrium, and is positive under Condition 2 .

The effect on $p_{H}^{*}$ of a change in $s_{L}$.

By Cramer's rule and using the definition of $\pi(\theta)$, we have:

$\frac{d p_{H}^{*}}{d s_{L}}=-\frac{\frac{p_{H}}{\Delta} f_{H}^{2}\left(2+\pi_{H}\right)+\frac{\Delta}{s_{L}}\left(1+\pi_{L}\right) f_{L}\left(\frac{p_{H}}{\Delta}\left(\theta_{H}-\theta_{L}\right) f_{H}^{\prime}+\left(\theta_{H}-\theta_{L}\right) f_{H}+\frac{p_{H}}{\Delta} f_{H}\right)}{f_{H}^{2}\left(2+\pi_{H}\right)+\frac{\Delta}{s_{L}}\left(1+\pi_{L}\right) f_{L}\left(\left(1+\pi_{H}\right) f_{H}+\frac{p_{L}}{\Delta} f_{H}^{\prime}\right)}$

where the RHS is evaluated at equilibrium. By using (A.2) for $\Delta / s_{L}$, (A.3) for $p_{L} / \Delta, p_{H} / \Delta=p_{L} / \Delta+\theta_{H}$, the definitions of $\eta$ for $f$, the definition of $\pi$ for $f^{\prime}$, and then equation $\left(5^{\prime}\right)$ for $\left(1-F_{L}\right)$, we obtain that at the price equilibrium equation (B.1) collapses to: 
$\frac{d p_{H}^{*}}{d s_{L}}=-\frac{\theta_{H}}{\eta_{H}} \frac{\left(2+\pi_{H}\right)\left(1-\eta_{H}\right)+\left(1+\pi_{L}\right) \eta_{L} \frac{2-\eta_{H}}{1-\eta_{L}}\left(\pi_{H}+\eta_{H}-\left(\pi_{H}+\eta_{H}-1\right) \frac{\theta_{L}}{\theta_{H}}\right)}{\left(2+\pi_{H}\right)\left(1-\eta_{H}\right)+\left(1+\pi_{L}\right) \eta_{L} \frac{2-\eta_{H}}{1-\eta_{L}}\left(\frac{2 \eta_{H}+\pi_{H}-1}{\eta_{H}}\right)}$

which under Conditions 2 and 3 is unambiguously negative.

The sign of $\left(\frac{d p_{L}^{*}}{d s_{L}}-\theta_{L}^{*}\right)$

Consider now $d p_{L}^{*} / d s_{L}$. By applying Cramer's rule and using the definition of $\pi(\theta)$, we obtain

$\frac{d p_{L}^{*}}{d s_{L}}=\theta_{L} \frac{\frac{\Delta}{s_{L}}\left(1+\pi_{L}\right) f_{L}\left[\left(1+\pi_{H}\right) f_{H}+\frac{p_{L}}{\Delta_{s}} f_{H}^{\prime}\right]-\frac{s_{L}}{\Delta} f_{H}^{2}\left(2+\pi_{H}\right)}{\frac{\Delta}{s_{L}}\left[\left(1+\pi_{L}\right) f_{L}\right]\left[\left(1+\pi_{H}\right) f_{H}+\frac{p_{L}}{\Delta_{s}} f_{H}^{\prime}\right]+f_{H}^{2}\left(2+\pi_{H}\right)}$

By using (A.2) for $\Delta / s_{L}$, (A.3) for $p_{L} / \Delta$, the definitions of $\eta$ for $f$, the definition of $\pi$ for $f^{\prime}$, and then equation ( $\left.5^{\prime}\right)$ for $\left(1-F_{L}\right)$, we obtain that at the price equilibrium equation (B.3) collapses to:

$\frac{d p_{L}^{*}}{d s_{L}}=\theta_{L} \frac{\left(1+\pi_{L}\right) \eta_{L} \frac{2-\eta_{H}}{1-\eta_{L}}\left(2 \eta_{H}+\pi_{H}-1\right)-\frac{\theta_{H}\left(1-\eta_{H}\right)^{2}}{\theta_{L}}\left(2+\pi_{H}\right)}{\left(1+\pi_{L}\right) \eta_{L} \frac{2-\eta_{H}}{1-\eta_{L}}\left(2 \eta_{H}+\pi_{H}-1\right)+\eta_{H}\left(2+\pi_{H}\right)\left(1-\eta_{H}\right)}$

Since under Condition 3 the value of the fraction in (B.4) is surely less than one, we get:

$\frac{d p_{L}^{*}}{d s_{L}}-\theta_{L}^{*}=\theta_{L}\left(\frac{\left(1+\pi_{L}\right) \eta_{L} \frac{2-\eta_{H}}{1-\eta_{L}}\left(2 \eta_{H}+\pi_{H}-1\right)-\frac{\theta_{H}\left(1-\eta_{H}\right)^{2}}{\theta_{L}}\left(2+\pi_{H}\right)}{\left(1+\pi_{L}\right) \eta_{L} \frac{2-\eta_{H}}{1-\eta_{L}}\left(2 \eta_{H}+\pi_{H}-1\right)+\eta_{H}\left(2+\pi_{H}\right)\left(1-\eta_{H}\right)}-1\right)<0$

where the RHS is evaluated at equilibrium.

\section{Appendix C. The Second Order Condition at the quality stage}

The proof that the Second Order Condition at the quality stage for firm $L$ is satisfied, relies on three preliminary steps.

STEP I. It is useful to calculate $\Psi^{\prime}\left(\theta_{H}\right)$ at $\Psi\left(\theta_{H}\right)=0$. By deriving equation (11) and recalling that $\theta_{L}=g\left(\theta_{H}\right)$ according to $\left(5^{\prime}\right)$, we get that at $\Psi\left(\theta_{H}\right)=$ 0 :

$\Psi^{\prime}\left(\theta_{H}\right)=\frac{\eta_{H}^{\prime} \eta_{L} \theta_{L}}{1-\eta_{L}} \frac{2-\eta_{H}}{1-\eta_{H}}+\left(1-\eta_{H}\right)\left(\frac{\partial p_{H L}}{\delta \theta_{H}}+\frac{\partial p_{H L}}{\delta \theta_{L}} g^{\prime}\left(\theta_{H}\right)+1\right)+$ 
$-\frac{\eta_{H}^{\prime}}{1-\eta_{L}} \eta_{L} \theta_{L}+\frac{2-\eta_{H}}{1-\eta_{L}}\left(\frac{\eta_{L} \theta_{L} \eta_{L}^{\prime}}{1-\eta_{L}}+\eta_{L}^{\prime} \theta_{L}+\eta_{L}\right) g^{\prime}\left(\theta_{H}\right)$

Collecting terms the above expression can be simplified into:

$\Psi^{\prime}=\frac{\eta_{H}^{\prime} \eta_{L} \theta_{L}}{\left(1-\eta_{L}\right)\left(1-\eta_{H}\right)}+\left(1-\eta_{H}\right)\left(\frac{\partial p_{H L}}{\delta \theta_{H}}+\frac{\partial p_{H L}}{\delta \theta_{L}} g^{\prime}\left(\theta_{H}\right)+1\right)+$

$+\frac{2-\eta_{H}}{1-\eta_{L}}\left(\frac{\theta_{L} \eta_{L}^{\prime}}{1-\eta_{L}}+\eta_{L}\right) g^{\prime}\left(\theta_{H}\right)$

Using now the definitions of $\eta(\theta)$ and $\pi(\theta)$, we can write:

$\eta_{L}^{\prime}=\frac{\eta_{L}}{\theta_{L}}\left(\pi_{L}+\eta_{L}\right) ; \quad \eta_{H}^{\prime}=\frac{\eta_{H}}{\theta_{H}}\left(\pi_{H}+\eta_{H}\right)$

which used into $\Psi^{\prime}\left(\theta_{H}\right)$ leads to:

$\Psi^{\prime}\left(\theta_{H}\right)=\frac{\theta_{L}}{\theta_{H}} \frac{\eta_{H}\left(\pi_{H}+\eta_{H}\right) \eta_{L}}{\left(1-\eta_{L}\right)\left(1-\eta_{H}\right)}+\left(1-\eta_{H}\right)\left(\frac{\partial p_{H L}}{\partial \theta_{H}}+\frac{\partial p_{H L}}{\partial \theta_{L}} g^{\prime}\left(\theta_{H}\right)+1\right)+$

$+\frac{2-\eta_{H}}{1-\eta_{L}} \eta_{L} \frac{\pi_{L}+1}{1-\eta_{L}} g^{\prime}\left(\theta_{H}\right)$

STEP II. Notice that at the price equilibrium the effects of changes in qualities on the value of $\theta_{H}$ and $\theta_{L}$ can be written as follows:

$$
\begin{aligned}
\theta_{L L} & \equiv \frac{\partial \theta_{L}^{*}}{\partial s_{L}}=\frac{1}{s_{L}}\left(p_{L L}-\theta_{L}\right)<0 \\
\theta_{H L} & \equiv \frac{\partial \theta_{H}^{*}}{\partial s_{L}}=\frac{1}{\Delta}\left(p_{H L}+\theta_{H}-p_{L L}\right)
\end{aligned}
$$

The sign of $\theta_{L L}$ can be easily established from (B.5). As far as $\theta_{H L}$ is concerned, we recall from Lemma 3 that for $\left(5^{\prime}\right)$ to be satisfied:

$$
\theta_{L L}=g^{\prime} \theta_{H L}
$$

so that, given $g^{\prime}>0, \theta_{H L}<0$.

SteP III. For future reference, it is useful to solve equation (8) in terms of $p_{L L}$. We recall that equation (8) is:

$$
p_{L L}\left(F_{H}-F_{L}\right)+p_{L} f_{H} \theta_{H L}=p_{L} f_{L} \theta_{L L}
$$


By using (C.4) for $\theta_{L L}$ and (C.3) for $\theta_{H L}$, it can be written as:

$$
p_{L L}=\frac{\left(\frac{p_{L}}{\Delta} f_{L} g^{\prime}-\frac{p_{L}}{\Delta} f_{H}\right)}{\left(F_{H}-F_{L}+\frac{p_{L}}{\Delta} f_{L} g^{\prime}-\frac{p_{L}}{\Delta} f_{H}\right)}\left(p_{H L}+\theta_{H}\right)
$$

Consider now the ratio at the RHS of the above expression. Recalling equations $\left(5^{\prime}\right)$ and (A.3), the expression for $g^{\prime}$ and the definitions of $\eta(\theta)$, we obtain:

$$
\frac{\left(\frac{p_{L}}{\Delta} f_{L} g^{\prime}-\frac{p_{L}}{\Delta} f_{H}\right)}{\left(F_{H}-F_{L}+\frac{p_{L}}{\Delta} f_{L} g^{\prime}-\frac{p_{L}}{\Delta} f_{H}\right)}=\frac{\left(1-\eta_{H}\right)\left(1+\pi_{H}-\pi_{L}\right)\left(1-\eta_{L}\right)}{\left(1-\eta_{H}+\eta_{L}\right)\left(1+\pi_{L}\right)+\left(1-\eta_{H}\right)\left(1-\eta_{L}\right)\left(1+\pi_{H}-\pi_{L}\right)}
$$

so that, using equation (10) for $\left(p_{H L}+\theta_{H}\right)$ we get:

$$
p_{L L}=-\theta_{L} \frac{\eta_{L}\left(2-\eta_{H}\right)\left(1+\pi_{H}-\pi_{L}\right)}{\left(1-\eta_{H}+\eta_{L}\right)\left(1+\pi_{L}\right)+\left(1-\eta_{H}\right)\left(1-\eta_{L}\right)\left(1+\pi_{H}-\pi_{L}\right)}
$$

Now we proceed to evaluate the SOC for firm $L$ at the quality stage. The first order derivative of the profits of firm $L$ with respect to quality (equation (8) in text) can be written as:

$$
\frac{\partial \Pi_{L}}{\partial s_{L}}=\frac{p_{L}}{\Delta} f_{H}\left(p_{H L}+\theta_{H}\right)+f_{L} \theta_{L}^{2}+\left(F_{H}-F_{L}-\frac{p_{L}}{\Delta} f_{H}-\theta_{L} f_{L}\right) p_{L L}
$$

Therefore, given equation (2), the second order derivative is:

$$
\begin{aligned}
\frac{\partial^{2} \Pi_{L}}{\partial s_{L}^{2}}= & \frac{\partial}{\partial s_{L}}\left(\frac{p_{L}}{\Delta} f_{H}\left(p_{H L}+\theta_{H}\right)\right)-p_{L L} \frac{\partial}{\partial s_{L}}\left(\frac{p_{L}}{\Delta} f_{H}\right)+ \\
& +\frac{\partial}{\partial s_{L}}\left(f_{L} \theta_{L}^{2}\right)+p_{L L} \frac{\partial}{\partial s_{L}}\left(F_{H}-F_{L}-\theta_{L} f_{L}\right)
\end{aligned}
$$

Using (C.2) in the first two terms, and the definition of $\pi_{L}$ in the last two terms of (C.6), we obtain:

$$
\begin{aligned}
& \frac{\partial^{2} \Pi_{L}}{\partial s_{L}^{2}}=\left(\left(2 p_{L L}+\frac{p_{L}}{\Delta}\right) f_{H}+\frac{p_{L}}{\Delta} f_{H}^{\prime}\left(p_{H L}+\theta_{H}-p_{L L}\right)\right) \theta_{H L}+ \\
& +f_{L}\left(\theta_{L}-p_{L L}\right)\left(1+\pi_{L}\right) \theta_{L L}+\frac{p_{L}}{\Delta} f_{H}\left(\frac{\partial p_{H L}}{\partial \theta_{H}}+1\right) \theta_{H L}+\frac{p_{L}}{\Delta} f_{H} \frac{\partial p_{H L}}{\partial \theta_{L}} \theta_{L L}
\end{aligned}
$$

which, using (A.3), (C.4), and the definition of $\eta(\theta)$, results into:

$\frac{\partial^{2} \Pi_{L}}{\partial s_{L}^{2}}=\left(\left(2 p_{L L}+\frac{p_{L}}{\Delta}\right) f_{H}+\frac{p_{L}}{\Delta} f_{H}^{\prime}\left(p_{H L}+\theta_{H}-p_{L L}\right)\right) \theta_{H L}+$ 


$$
\begin{aligned}
& f_{L}\left(\theta_{L}-p_{L L}\right)\left(1+\pi_{L}\right) g^{\prime} \theta_{H L}+\left(1-F_{H}\right)\left(1-\eta_{H}\right)\left(\frac{\partial p_{H L}}{\partial \theta_{H}}+1\right) \theta_{H L}+ \\
& +\frac{\partial p_{H L}}{\partial \theta_{L}} g^{\prime} \theta_{H L}
\end{aligned}
$$

Consider now (C.1) . By multiplying both sides by $\theta_{H L}$ and rearranging terms, it can be rewritten as:

$$
\begin{aligned}
& \left(1-\eta_{H}\right)\left(\left(\frac{\partial p_{H L}}{\partial \theta_{H}}+1\right) \theta_{H L}+\frac{\partial p_{H L}}{\partial \theta_{L}} g^{\prime} \theta_{H L}\right)= \\
& =\theta_{H L} \Psi^{\prime}\left(\theta_{H}\right)-\left(\frac{\theta_{L}}{\theta_{H}} \frac{\eta_{H}\left(\pi_{H}+\eta_{H}\right) \eta_{L}}{\left(1-\eta_{L}\right)\left(1-\eta_{H}\right)} \theta_{H L}+\frac{2-\eta_{H}}{1-\eta_{L}} \eta_{L} \frac{\pi_{L}+1}{1-\eta_{L}} g^{\prime} \theta_{H L}\right)
\end{aligned}
$$

Using (C.1') into (C.7) we obtain:

$$
\begin{aligned}
& \frac{\partial^{2} \Pi_{L}}{\partial s_{L}^{2}}=\left(1-F_{H}\right) \theta_{H L} \Psi^{\prime}\left(\theta_{H}\right)+f_{L}\left(\theta_{L}-p_{L L}\right)\left(1+\pi_{L}\right) g^{\prime} \theta_{H L}+ \\
& +\left(\left(2 p_{L L}+\frac{p_{L}}{\Delta}\right) f_{H}+\frac{p_{L}}{\Delta} f_{H}^{\prime}\left(p_{H L}+\theta_{H}-p_{L L}\right)\right) \theta_{H L}+ \\
& -\left(1-F_{H}\right)\left(\frac{\theta_{L} \eta_{H}\left(\pi_{H}+\eta_{H}\right) \eta_{L}}{\theta_{H}\left(1-\eta_{L}\right)\left(1-\eta_{H}\right)} \theta_{H L}+\frac{2-\eta_{H}}{1-\eta_{L}} \eta_{L} \frac{\pi_{L}+1}{1-\eta_{L}} g^{\prime} \theta_{H L}\right)
\end{aligned}
$$

The first term in (C.8) is negative, since $\theta_{H L}<0$ (see Step II of this Appendix) and $\Psi^{\prime}>0$ at equilibrium. In the sequel we prove that the sum of the remaining terms is equal to zero at equilibrium. By using repeatedly equations (5'), (10), (A.3) as well as the expression for $g^{\prime}$ and the definitions of $\eta(\theta)$ and $\pi(\theta)$, tedious algebra shows that this amounts to proving that:

$$
\begin{aligned}
& 2 p_{L L} \frac{\eta_{H}}{\theta_{H}}+\left(1-\eta_{H}\right)+\left(1-\eta_{H}\right) \frac{1}{\theta_{H}}\left(\pi_{H}-1\right)\left(p_{H L}+\theta_{H}\right)+ \\
& -\left(1-\eta_{H}\right) \frac{1}{\theta_{H}}\left(\pi_{H}-1\right) p_{L L}+\frac{\eta_{H}}{\theta_{H}}\left(2+\pi_{H}\right) \theta_{L}-p_{L L} \frac{\eta_{H}}{\theta_{H}}\left(2+\pi_{H}\right)+ \\
& -\left(\frac{\theta_{L}}{\theta_{H}} \frac{\eta_{H}\left(\pi_{H}+\eta_{H}\right) \eta_{L}}{\left(1-\eta_{L}\right)\left(1-\eta_{H}\right)}+\eta_{H} \frac{\left(2+\pi_{H}\right)}{1-\eta_{L}} \frac{\theta_{L}}{\theta_{H}}\right)=0
\end{aligned}
$$

By substituting $p_{H L}+\theta_{H}$ from equation (10), collecting terms, and substituting for $p_{L L}$ from equation (C.5), this expression can be transformed into: 


$$
\begin{aligned}
& \left(1-\eta_{H}\right)+ \\
& -\frac{\theta_{L} \eta_{L}\left(2-\eta_{H}\right)}{\theta_{H}\left(1-\eta_{L}\right)\left(1-\eta_{H}\right)}\left(\frac{\left(1-\eta_{H}+\eta_{L}\right)\left(1+\pi_{L}\right)\left(2 \eta_{H}+\pi_{H}-1\right)+\eta_{H}\left(1-\eta_{L}\right)\left(1-\eta_{H}\right)\left(1+\pi_{H}-\pi_{L}\right)}{\left(1-\eta_{H}+\eta_{L}\right)\left(1+\pi_{L}\right)+\left(1-\eta_{L}\right)\left(1-\eta_{H}\right)\left(1+\pi_{H}-\pi_{L}\right)}\right)=0
\end{aligned}
$$

The term $\theta_{L} \eta_{L}\left(2-\eta_{H}\right) / \theta_{H}\left(1-\eta_{L}\right)\left(1-\eta_{H}\right)$ can again be substituted from equation (10), so that we get:

$$
\begin{aligned}
& \left(1-\eta_{H}\right)+ \\
& +\left(\frac{p_{H L}}{\theta_{H}}+1\right)\left(\frac{\left(1-\eta_{H}+\eta_{L}\right)\left(1+\pi_{L}\right)\left(2 \eta_{H}+\pi_{H}-1\right)+\eta_{H}\left(1-\eta_{L}\right)\left(1-\eta_{H}\right)\left(1+\pi_{H}-\pi_{L}\right)}{\left(1-\eta_{H}+\eta_{L}\right)\left(1+\pi_{L}\right)+\left(1-\eta_{L}\right)\left(1-\eta_{H}\right)\left(1+\pi_{H}-\pi_{L}\right)}\right)=0
\end{aligned}
$$

Now, we deal with the term $\left(p_{H L} / \theta_{H}\right)+1$. At equilibrium, given (B.2) and (10):

$$
\begin{aligned}
& \left(\frac{p_{H L}}{\theta_{H}}+1\right)= \\
& -\left(1-\eta_{H}\right) \frac{\left(1+\pi_{L}\right) \eta_{L} \frac{2-\eta_{H}}{1-\eta_{L}}+\left(1-\eta_{H}\right)\left(2+\pi_{H}\right)}{\left(1+\pi_{L}\right) \eta_{L} \frac{2-\eta_{H}}{1-\eta_{L}}\left(2 \eta_{H}+\pi_{H}-1\right)+\left(1-\eta_{H}\right)\left(2+\pi_{H}\right) \eta_{H}+\left(1+\pi_{L}\right)\left(\pi_{H}+\eta_{H}-1\right)\left(1-\eta_{H}\right)}
\end{aligned}
$$

which, substituted into (C.9) yields:

$$
1-\frac{\left(1-\eta_{H}+\eta_{L}\right)\left(1+\pi_{L}\right)\left(2 \eta_{H}+\pi_{H}-1\right)+\eta_{H}\left(1-\eta_{L}\right)\left(1-\eta_{H}\right)\left(1+\pi_{H}-\pi_{L}\right)}{\left(1+\pi_{L}\right) \eta_{L}\left(2-\eta_{H}\right)\left(2 \eta_{H}+\pi_{H}-1\right)+\left(1-\eta_{H}\right)\left[2+\pi_{H}\right] \eta_{H}\left(1-\eta_{L}\right)+\left(1+\pi_{L}\right)\left(\pi_{H}+\eta_{H}-1\right)\left(1-\eta_{H}\right)\left(1-\eta_{L}\right)}=0
$$

which is actually true. Therefore equation (C.8) collapses to

$$
\frac{\partial^{2} \Pi_{L}}{\partial s_{L}^{2}}=\left(1-F_{H}\right) \theta_{H L} \Psi^{\prime}\left(\theta_{H}\right)<0
$$




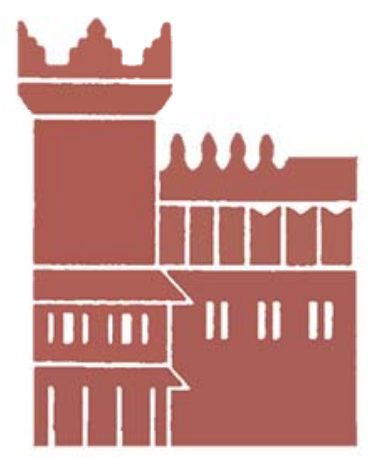

Alma Mater Studiorum - Università di Bologna DEPARTMENT OF ECONOMICS

Strada Maggiore 45

40125 Bologna - Italy

Tel. +39051 2092604

Fax +390512092664

http://www.dse.unibo.it 